\title{
Level of education and preferred language of informed consent for clinical research in a multi-lingual community
}

\author{
Grace Muzanyi ${ }^{1}$, Isaac Sekitoleko ${ }^{1}$, John L Johnson ${ }^{1,2}$, Jane Lunkuse ${ }^{1}$, Gladys \\ Nalugwa ${ }^{1}$, Joanita Nassali ${ }^{1}$, David Kaawa Mafigiri ${ }^{1,3}$
}

1. Uganda-Case Western Reserve University Research Collaboration, Kampala, Uganda.

2. Case Western Reserve University School of Medicine and University Hospitals Cleveland Medical Center, Cleveland, Ohio, USA.

3. Center for Social Science Research on AIDS, School of Social Sciences. Makerere University, Kampala, Uganda.

\begin{abstract}
:
Background: Low education levels and language barriers present challenges in obtaining informed consent for clinical research.

Objective: To describe and correlate the association between the level of education and the participant's preferred language of consent.

Design: Descriptive-analytical cross-sectional study.

Participants: Adults being consented for participation in tuberculosis(TB) research studies in an East African community with varying levels of education.

Procedures: We analyzed data on demographic and educational characteristics collected from adults being consented for participation in TB studies .Only participants who could understand and speak Luganda (the main local language) or English ( the official language of Uganda) were included in this analysis.

Results: A total of 523 participants were consented between April 2015 and December 2017 and included in this analysis; 250 below Senior four (<11yrs of education), 114 senior four (at 11yrs of education), 73 senior five-senior six (12-13yrs of education) and 86 beyond senior six ( $>13 \mathrm{yrs}$ of education). We noted that the preference for English rises with the rising levels of education and peaked at beyond senior six $(83 \% \mathrm{Vs} 17 \%, \mathrm{OR}=49,95 \% \mathrm{CI}: 22.8-106.3, \mathrm{p}<0.001)$. Participants below senior four preferred Luganda Vs senior four and above $(\mathrm{OR}=16.9,95 \% \mathrm{CI}: 9.9-28.8, \mathrm{p}<0.001)$

Conclusion: Rising education levels of participants were associated with preference for English language usage during initial consent for clinical research studies.

Keywords: Level of education; preferred language; informed consent; multi-lingual community.

DOI: https://doi.org/10.4314/ahs.v20i2.51

Cite as: Muzanyi G, Sekitoleko I, Johnson JL, Lunkuse J, Nalugwa G, Nassali J, et al. Level of education and preferred language of informed consent for clinical research in a multi-lingual community. Afri Health Sci. 2020; 20(2): 955-959. https:// doi.org/10.4314/ abs.v20i2.51
\end{abstract}

\section{Introduction}

Current guidelines for clinical research stipulate that research procedures and consent documents should

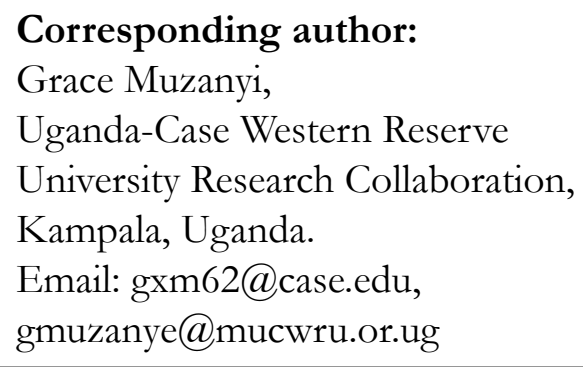

be presented in a language understood by research participants ${ }^{1,2,3}$. Informed consent is a process of information exchange that requires dialogue between the participant and the researcher ${ }^{1,4}$. With varying literacy levels; we don't understand the ease of this dialogue, ease of comprehension ,how people make consent language choices ${ }^{5}$ and what influences their choices. Consequently with varying levels of literacy; challenges with comprehension and difficulties in disclosure that include giving participants the information they need can arise ${ }^{4}$. 
Higher literacy levels have been associated with increased comprehension and satisfaction with the informed consent process ${ }^{6}$ however, there is limited literature on how the level of education influences the choice of language for informed consent.

According to UNESCO, the adult literacy rate in Uganda in 2012 was 70\%. Literacy was higher among younger persons less than 24 years-old and was greater in males $(79 \%)$ than females $(62 \%)$. Over 40 languages are spoken in Uganda but English is the official language ${ }^{7}$. In Uganda, schools at all levels conduct lessons in English. In the Kampala area, the most common languages are Luganda and English. Senior secondary school level four (O-level)is perceived as the education level at which one is able to read, write and speak English in Uganda.

Sub-Saharan Africa is a multi-lingual region ${ }^{15}$. In multi-lingual settings, the local language of consent adapted by researchers is determined by the dominant ethnic group (s) keeping the education level constant.In central Uganda, Luganda is the most widely spoken local language given that the baganda tribe is the most dominant group in this region ${ }^{14}$. Some research participants may prefer the local language to English regardless of their level of education.

We hypothesized that participants would prefer to consent in their local (mother) language regardless of the level of education ${ }^{13}$ as opposed to the English language. We therefore sought to study whether there is any association between the level of education (literacy) and the choice of language for informed consent. Identifying the preferred language of consent would ease recruitment of research participants in multi-lingual communities as well as maintaining the validity and integrity of the informed consent process.

\section{Methods}

We conducted a cross-sectional analysis of informed consent data collated from adults with suspected pulmonary tuberculosis or their adult contacts who were consented for screening for possible enrollment in one or more TB epidemiologic or prevention and treatment research studies being conducted at the Uganda-Case Western Reserve University Research Collaboration Project Clinic,an established research unit located at Mulago Hospital, Kampala, the largest teaching hospital in Uganda. Potential participants were referred to the research Clinic from the wards and clinics at Mulago Hospital as well as from health facilities in the com- munity located within a $30 \mathrm{~km}$ radius. Potential participants were given general information about TB and the ongoing TB research studies at the clinic by trained health educators and nurse counselors who were fluent in both Luganda and English.Interested volunteers were then asked to give informed consent for general screening for potential participation in the ongoing TB research studies .Screening procedures included collection of demographic information,medical history and physical examination, chest radiography,HIV testing,full blood count,liver and renal function tests,urine pregnancy testing (females only) and sputum examination. General screening procedures and the English and Luganda consent forms were reviewed and approved by both Ugandan and US institutional review boards. The Luganda version of the consent document was translated and back translated from the English version to assure accuracy. Participants individually chose whether to consent using the English or Luganda version of the consent form during the process. For illiterate persons, the consent form was read to them in the language of their choice in the presence of an independent witness. All participants had an opportunity to internalize and reflect on the information, and ask questions.

Volunteers who gave informed consent for general screening and completed initial evaluation were eligible for this analysis. Individuals who did not speak Luganda or English and persons who had participated in earlier research studies were excluded

\section{Statistical methods}

The data was analyzed using Stata v12.012.We used the chi-square analysis to determine differences in the proportion of participants that preferred a specific language at each level of education. Given the age distribution for education in Uganda with the highest levels of literacy being found among persons less than $24 \mathrm{yrs}^{8}$, age was divided into 3 categories:18-25,26-35\&>35yrs. Per Ugandan education curriculum ${ }^{8}$, there are four main levels(below senior 4, senior four,senior five to senior six and above senior six). The education levels were defined in years of attending school: Below senior four is equivalent to less than 11 years of education,senior four equates to 11 years of education,senior five -six equates to 12-13 years of education and beyond senior six equates to more than 13 years of education. We therefore stratified the education level into four categories: below senior four, at senior four,senior five to senior six and above senior six/tertiary institution of learning. The odds ratio, 95\% CI and the corresponding 
P-value was the effect measure. All the P-values were two sided. To further understand the differences in language preference across different levels of education, we used multivariate analysis to determine the independent contribution of patient factors (Age, gender, employment, marital status\&alcoholism).

\section{Results \\ Study population}

In total 1189 were selected for generic screening between 24-April-2015 and 31 December 2017 of which 523 consented. Of the total (523) that consented: 250 were below Senior four, 114 were senior four, 73 were senior five to senior six and 86 were beyond senior six. A total of 666 were excluded and the main reasons for exclusion were: Living outside the study area of $30 \mathrm{Km}$ radius $(\mathrm{N}=50)$, not able to produce $\operatorname{sputum}(\mathrm{N}=40)$,
Ineligible for any $\mathrm{TB}$ study $(\mathrm{N}=59)$, previous TB treatment $(\mathrm{N}=38)$, too $\operatorname{sick}(\mathrm{N}=34)$ \& other reasons $(\mathrm{N}=445)$ : mentally incompetent, not of consenting age, too busy to participate, not interested in research, unable to speak either English or Luganda, wants treatment without any further delays, fears blood draws. A total of 314 Luganda and 209 English consent forms were documented and included in this analysis. The baseline characterisitics of participants who consented in English were different from the Luganda language consenting participants(table 1). Some of the differences in the baseline characteristics could be attributed to the differnces in the levels of education(table 2). The Luganda speakers were $82 \%$ (258/314) baganda but the English speakers were more diverse with regard to their ethnicity. All the informed consents were 100\% (523/523) documented on an outpatient basis. No inpatients were considered for this study.

Table 1 Characteristics of study participants

\begin{tabular}{|c|c|c|c|c|c|}
\hline & $\begin{array}{l}\text { English } \\
\text { language } \mathrm{n}(\%) \\
(\mathrm{N}=209)\end{array}$ & $\begin{array}{l}\text { Luganda } \\
\text { language } n(\%) \\
(\mathrm{N}=60)\end{array}$ & $\begin{array}{l}\text { Total } \\
(\mathrm{N}=523)\end{array}$ & $\begin{array}{l}\text { Univariate } \\
\text { OR }(95 \% \mathrm{Cl})\end{array}$ & $\begin{array}{l}\text { Multivariate } \\
\text { OR }(95 \% \mathrm{CI})\end{array}$ \\
\hline Male sex (n,\%) & $137(39)$ & $218(61)$ & 355 & $0.84(0.58-1.21)$ & $1.04(0.63-1.73)$ \\
\hline \multicolumn{6}{|c|}{ Age (years, IQR, range) } \\
\hline $18-25$ & $114(56)$ & $90(44)$ & 204 & -- & -- \\
\hline $26-35$ & $61(36)$ & $108(64)$ & 169 & $0.45(0.29-0.68)$ & $0.36(0.21-0.61)$ \\
\hline$>35$ & $34(23)$ & $116(77)$ & 150 & $0.23(0.14-0.37)$ & $0.31(0.17-0.57)$ \\
\hline Unemployed & $77(51)$ & $73(49)$ & 150 & $1.93(1.31-2.82)$ & $1.37(0.81-2.33)$ \\
\hline Consumes alcohol & $36(38)$ & $60(63)$ & 96 & $0.88(0.56-1.39)$ & $1.99(1.08-3.67)$ \\
\hline \multicolumn{6}{|l|}{ Education level } \\
\hline Below S4 & $31(12)$ & $219(88)$ & 250 & -- & -- \\
\hline S4 & $64(56)$ & $50(44)$ & 114 & $9.04(5.33-15.33)$ & $8.97(5.16-15.5)$ \\
\hline S5-S6 & $43(59)$ & $30(41)$ & 73 & $10.13(5.56-18.43)$ & $8.79(4.64-16.63)$ \\
\hline Greater than S6 & $71(83)$ & $15(17)$ & 86 & $33.44(17.08-65.48$ & $36.53(17.98-74.19)$ \\
\hline Total & $209(40)$ & $314(60)$ & 523 & & \\
\hline
\end{tabular}

\section{Primary outcome}

The preference for English peaked at beyond senior six (83\%Vs17\%, OR=49, 95\%CI: 22.8-106.3,p<0.001). Overall people below senior four preferred Luganda compared to senior four and above $(\mathrm{OR}=16.9,95 \% \mathrm{CI}$ : 9.9-28.8, $\mathrm{p}<0.001)$.In addition, multivariate analysis adjusting for participant ethnic group, None Baganda ethnic groups preferred English compared to the Baganda $(66 \% \mathrm{Vs} 36 \%$, OR=7.8, 95\%CI: 3.7-16.4, $\mathrm{p}<0.001)$. This could be attributed to the fact the tested local lan- guage is primarily for the Baganda ethnic group. No further follow up beyond the initial consent to establish continued study stay in relation to the language of consent was conducted. We could therefore not establish if the preferred language of consent was associated with study participant retention. We did not stratify our analysis by other local languages since the study was done in central Uganda where Luganda is the main local language spoken and stratification by each local language would create too many subgroups that would lead to multiplicity of data. 


\begin{tabular}{|c|c|c|c|c|c|c|}
\hline \multirow[b]{3}{*}{ Variables } & \multicolumn{2}{|c|}{ Consent Language } & \multirow[b]{3}{*}{ P- } & \multirow[b]{3}{*}{ Value } & \multirow[b]{3}{*}{ OR } & \multirow[b]{3}{*}{$95 \% \mathrm{CI}$} \\
\hline & English & Luganda & & & & \\
\hline & N (\%) & $\mathrm{N}(\%)$ & & & & \\
\hline \multicolumn{7}{|c|}{ Education Level } \\
\hline Below S4 & $31(12)$ & $219(88)$ & & & $\ldots$ & - \\
\hline S4\&above & $178(56)$ & $95(44)$ & $<0.001$ & & 16.9 & $9.9-28.8$ \\
\hline \multicolumn{7}{|l|}{ Age group } \\
\hline $18-25$ & $114(55)$ & $90(44)$ & & & --- & - \\
\hline $26-35$ & $61(36)$ & $108(64)$ & $<0.001$ & & 0.38 & $0.23-0.63$ \\
\hline$>35$ & $34(23)$ & $116(77)$ & $<0.001$ & & 0.31 & $0.17-0.56$ \\
\hline
\end{tabular}

\section{Discussion}

In clinical research, informed consent is mandatory and comprehension of the passed on information to participants is key. Our study showed that a research participant's choice of the consent language is associated with the level of education. We have not identified any other study in the current published literature addressing a similar question for comparison with our findings. Our results show a shift of consent language preference from Luganda to English as the literacy level rises. The relationship between the preferred language of consent and level of education was not affected by gender however; preference for English was associated with being younger (18-25yrs old). This could be attributed to the scenario of age group 18-25 yrs being a higher education cohort where majority are attending high institutions of learning. With English being the official language at school in Uganda, the level of comprehension and ease to speak the English language is higher in this group. We as well found that preference for Luganda was associated with alcohol consumption on multivariate analysis. This could be attributed to the fact that Luganda preferring participants are likely to be less educated and therefore less knowledgeable about the dangers of excessive alcohol consumption in comparison to the English speaking group who are likely to be educated and therefore knowledgeable about the dangers of alcohol consumption. Garcia and colleagues ${ }^{6}$ showed that age and education are key to comprehension of information passed on to patients before surgery but did not assess the impact of the diffeent education levels to the comprehension of information. This is one study in the currently published literature that came close to assessing the impact of education on the consent process but however this study didn't objectively assess consent language choice and level of education. Our study findings have made some clari- fication that the level education is associated with the choice of the consent language which significantly impacts on comprehension. Studies have shown that individuals with low literacy and low English proficiency are always neglected in clinical research ${ }^{16}$. Our study findings could be a highlight on how to go about enrolling low literacy and low English proficiency populations through exploring the association between the level of education and choice of the consent language. By emphasizing translation of the consent forms into local languages with the participant's education level in perspective could be one way of enhancing the recruitment of low literacy and limited English proficiency participants.

The differences we found in the proportions of language preference with rising levels of education were significant and may suggest disparities in the informed consent comprehension among consenting prospective clinical research participants in relation to their level of education. This might have implications for clinical research conduct in communities with varying levels of literacy. Ethics committees recommend translation and back translation of consent documents between languages however per this study findings, it may imply putting less emphasis on translation and back translation between English \& local languages for research involving higher institutions of learning. For the local language (Luganda), not all participants that selected this language were from this ethnic group of the baganda however, they were able to fluently speak and understand Luganda. This could imply that for research involving a multi-lingual community with a dominant language, emphasis on translation and back translation should focus on the dominant language as opposed to all the different languages in that community. This could expedite participant accrual in clinical research studies.

African Health Sciences Vol 20 Issue 2, June, 2020 
Our study also found a relatively low level of literacy $(52 \%)$ compared to the current national average for Uganda $(70 \%) 8$. This could be attributed to our study only enrolling adults ( $\geq 18$ yrs) compared to the national average which captures $\geq 15 \mathrm{yrs}^{4}$

\section{Limitations}

It was conducted in an urban population which raises questions as to whether the study findings could be extrapolated to a rural population since most of the less educated tend to reside in the rural areas.In addition our study was conducted in one ethnic language of luganda.There could be cross cultural differences across different tribes and it raises questions whether our findings can be extrapolated to some of the other ethnic groups.

The strength for our study is that it was conducted in a multi-lingual setting and the results of our study could be extrapolated to many different tribes across multitribal Africa with more than 2000 different languages spoken $^{15}$.

\section{Recommendation}

For policy per our study findings is that research involving higher institutions of learning may require less emphasis of translation and back translation of the consent documents between the original research language and the local languages. These study findings are crucial to the approach to clinical research study accrual and comprehension of the informed consent process.

\section{References}

1. The HelsinkiDeclartion oftheWorld Medical Association(WMA). Ethical principles of medicalresearc involving human subjects].: Pol Merkur Lekarski. 2014 May; 36(215): 298-301: PMID:24964504

2. Uganda National Council for Science and Technology (UNCST). National Guidelines for Research involving Humans as Research Participants. Kampala, Uganda: UNCST; 2014

3. Uganda National CouncilforScienceandTechnologyUNCST).ResearchRegistrationandClearance:Policy and Guidelines. Kampala, Uganda: UNCST; 2016.

4. Flory J, Emanuel E. Interventions to improve research participants' understanding in informed consent for research: a systematic review. JAMA. 2004;292(13):1593-601.
5. Rozovsky F. Consent to Treatment: A Practical Guide. 2ed.Boston, MA: Little, Brown and Company;1990.

6. Garcia-Garcia EM, Sanchez-Sabater B, Medrano-Morte I, Luna-Maldonado A. Socio-demographic factors affecting the comprehension of clinical information by inpatients undergoing trauma surgery. Rev Esp Cir Ortop Traumatol. 2019

7. Schalit N. English rules in Uganda, but local languages shouldn't be sidelined. The Conversation. 2015.

8. Uganda Bureau of Statistics. National population and housing census 2014. Mainreport.Kampala: Uganda Bureau of Statistics; 2016. ix, 86 pages $p$.

9. Schenker Y, Wang F, Selig SJ, Ng R, Fernandez A. The impact of language barriers on documentation of informed consent at a hospital with on-site interpreter services. J Gen Intern Med. 2007;22 Suppl 2:294-9

10. AbdelRahmanSM.FacilitatingInformedPermission/ Assent/ConsentinPediatricClinicalTrials. Paediatr Drugs. 2019;21(4):205-14

11.Breese PE, Burman WJ, Goldberg S, Weis SE. Education level, primary language, and comprehension of the informed consent process. J Empir Res Hum Res Ethics. 2007;2(4):69-79.

12. StataCorp. 2011. Stata 12 Base Reference Manual. College Station, TX: Stata Press

13. Patients' understanding of telemedicine terms required for informed consent when translated into Kiswahili BMC Public Health. 2018 May 3;18(1):588. doi: 10.1186/s12889-018-5499-1:Odhiambo R1, Mars M29.PMID:28185201

14."WhatLanguagesAreSpokeninUganda?":Sawe,BenjaminElsha:WorldAlas,Aug.11,2017,worldatlas.com/articles/what-languages-are-spoken-in-uganda.html.

15. Multilingualism in Sub-Saharan Africa: B. Rotimi Badejo: Africa Media Review Vol. 3 No. 2. 1989 ®AfricanCouncilonCommunicationEduction:https://pdfs. semanticscholar.org/e0e4/b62fd1c43b23d507564affbbfca69da77b63.pdf

16. Lee JS, Pérez Stable EJ, Gregorich SE, Crawford MH, Green A, Livaudais Toman J, Karliner LS: Increased Access to Professional Interpreters in the Hospital Imroves Informed Cosent for Patients with Limited English Proficiency.: J Gen Intern Med. 2017Aug; 32(8):863870.Doi:10.1007/s1160601739834.Epub 2017 Feb 9.PMID:28185201 